\title{
OIIIA \\ AIAA 01-4557
}

Autonomous System for MISSE Temperature Measurements

G. A. Harvey, T. J. Lash, W. H. Kinard, K. Bull, and F. de Geest

NASA Langley Research Center

Hampton, VA 23681-2199

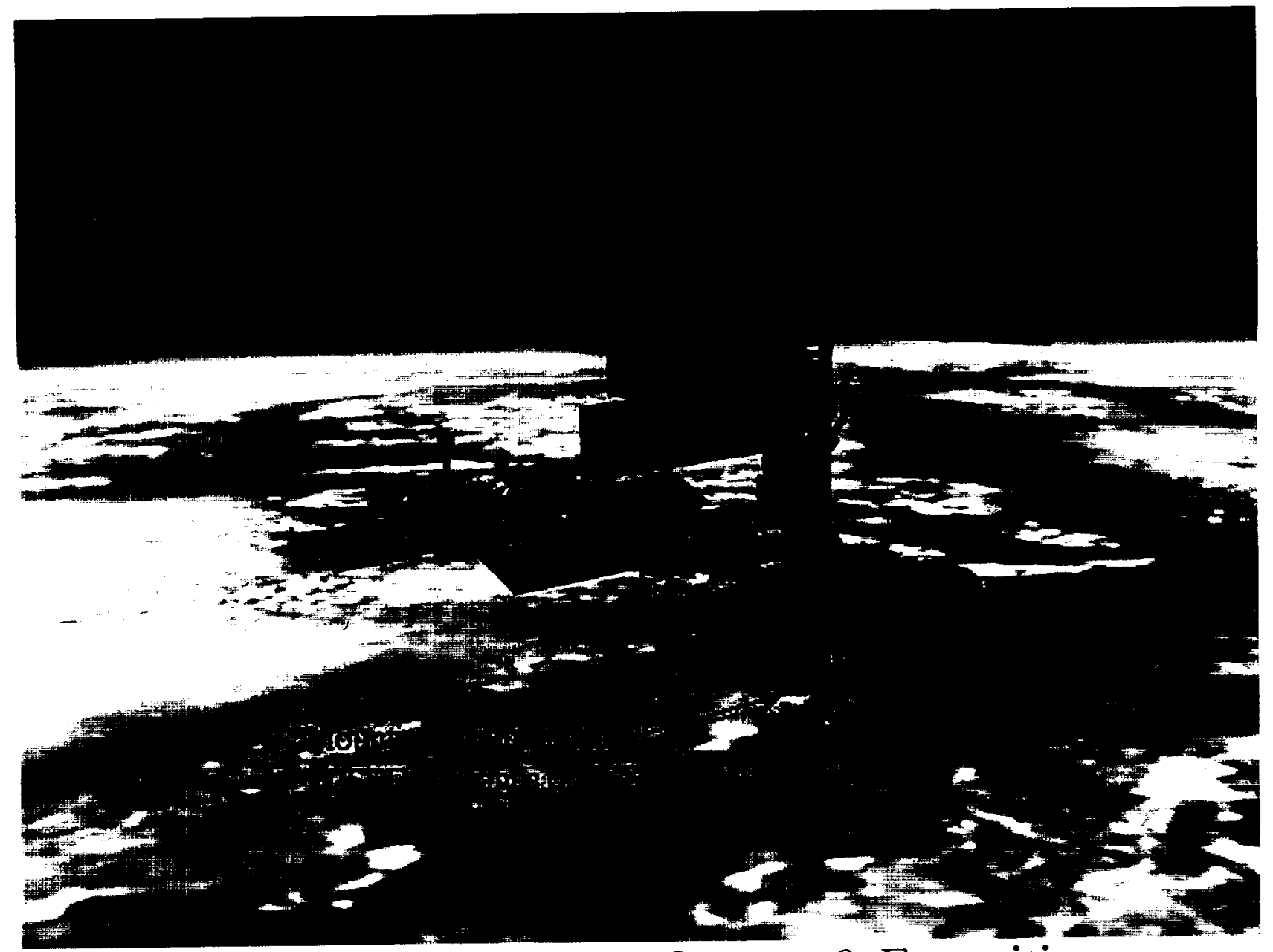

AIAA Space 2001 Conference \& Exposition

28-30 August 2001

Albuquerque, NM

For permission to copy or to republish, contact the copyright owner named on the first page.

For ALAA-held copyright, write to AIAA Permissions Department,

1801 Alexander Bell Drive, Suite 500, Reston, VA, 20191-4344. 
AIAA 01-4557

\title{
Autonomous System for MISSE Temperature Measurements
}

\author{
G. A. Harvey", T. J. Lash', W. H. Kinard ${ }^{*}$, K. Bull ${ }^{a}$, and F. de Geest ${ }^{s}$ \\ NASA Langley Research Center \\ Hampton, VA 23681-2199
}

\begin{abstract}
The Materials International Space Station Experiment (MISSE) is scheduled to be deployed during the summer of 2001 . This experiment is a cooperative endeavor by NASA-LaRC, NASA-GRC, NASA MSFC, NASA-JSC, the Materials Laboratory at the Air Force Research Laboratory, and the Boeing Phantom Works. The objective of the experiment is to evaluate performance, stability, and long term survivability of materials and components planned for use by NASA and DOD on future LEO, synchronous orbit, and interplanetary space missions. Temperature is an important parameter in the evaluation of space environmental effects on materials
\end{abstract}

\section{INTRODUCTION}

The International Space Station (ISS) (Figure 1) is a key element of the NASA Human Exploration and Development of Space Enterprise. The ISS will expand our knowledge and help bring the benefits of space to Earth. The first element of the ISS was launched in November 1998. The ISS became inhabited in October 2000. The ISS is scheduled for completion in the 2005-2006 timeframe

The Long Duration Exposure Facility (LDEF) and the Mir Environmental Effects Payload (MEEP) utilized hardware returned from low-Earth-orbit (LEO) to study and measure space and spacecraft-induced environmental effects. A section of a solar array relumed from the Mir space station has also been extensively studied for power degradation. micrometeoroid, and contamination effects

\footnotetext{
"Research Scientist. Test and Devielopment Branch, Srstems Engineering Competency

Electrical Engineering Technician, Test and Development Branch. Systems Engineering Competencr

"Materials Scientist, Environmental Interactions Branch. Research and Technology Competency.

President, Verited Instruments Inc.

SSales Manager, Veriteq Instruments Inc

Copyright $a 200]$ by the American Institute of Aeronautics and Astronautics, Inc. No copvright is asserted in the United States unler Title 17, US Code. The US Government has a royalty-free license to exercise all rights under the copyright claimed hercin for gosernmental purposes. All other copyrights are reserved by the swner.
}

Numerous papers, including references $1-6$ have been written on analyses of the returned hardware. The LDEF had more than six years of space exposure. the MEEP hardware had more than one year of space exposure, and the returned Mir solar array section had more than ten years of space exposure.

The space-exposed materials of the MEEP experiment were housed in suitcase-like containers (Figure 2) for transporting to and from Mir via the Shuttle. Four of these Payload Experiment Containers $\because$ (PECs) were clamped onto the handrails of the MirOrbiter docking module. These are being refurbished and are scheduled for early space exposure experiments on the ISS. These refurbished PECs (Figure 3) constitute the flight hardware of the Materials International Space Station Experiment (MISSE).

MISSE is being developed as an element of the Engineering Research and Technology Program at Johnson Space Center as an innovative technical concept to expand the utilization of the ISS. It is a cooperative endeavor by the NASA Langley Research Center, NASA Glenn Research Center, NASA Marshall Space Flight Center, NASA Johnson Space Center, the Materials Laboratory at the Air Force Research Laboratory, and the Boeing Phantom Works. The objective of MISSE is to evaluate performance, stability, and long term survivability of materials and components planned for use by NASA and DOD on future LEO, synchronous orbit, and interplanetary space missions.

Over 700 materials specimens (Figure 4) are contained in PECs 1 and 2. Temperature is a key parameter in space environmental effects on materials and hardware. External hardware temperatures are complex functions of angle of the hardware surface to the sun, the hardware absorptivity and emissivity, thermal mass, conductivity to adjacent hardware and previous thermal history of the hardware. The angle of the hardware surface to the sun is a complex function of the spacecraft position in orbit (which continuously changes during an orbit), the plane of the orbit with respect to the sun (which changes or precesses from orbit to orbit and effects time-in shadow or seasonal changes), location and orientation of the hardware on the spacecraft, and orientation of the spacecraft (attitude) with respect to the orbit. Computations of hardware temperature histories are 

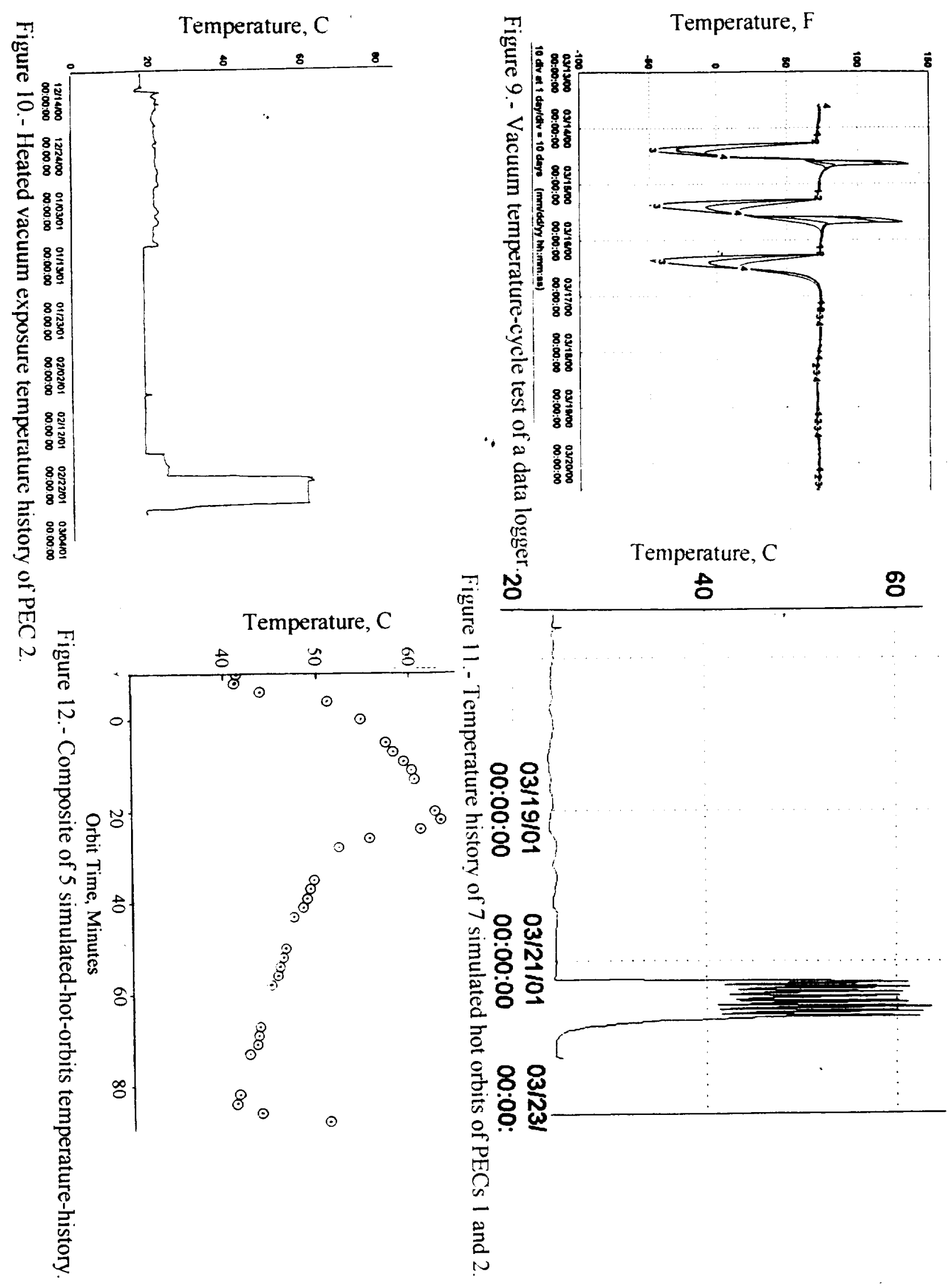

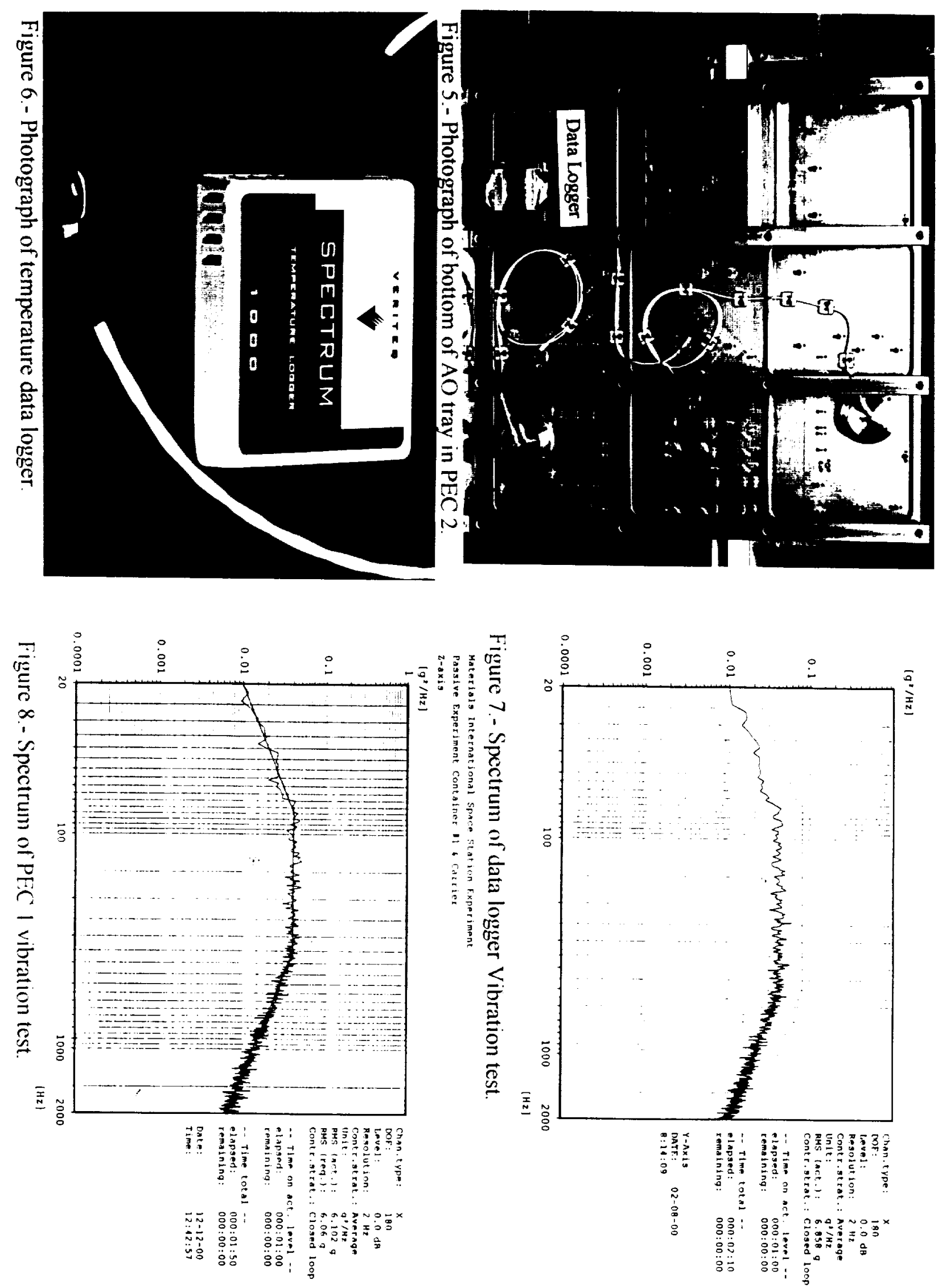

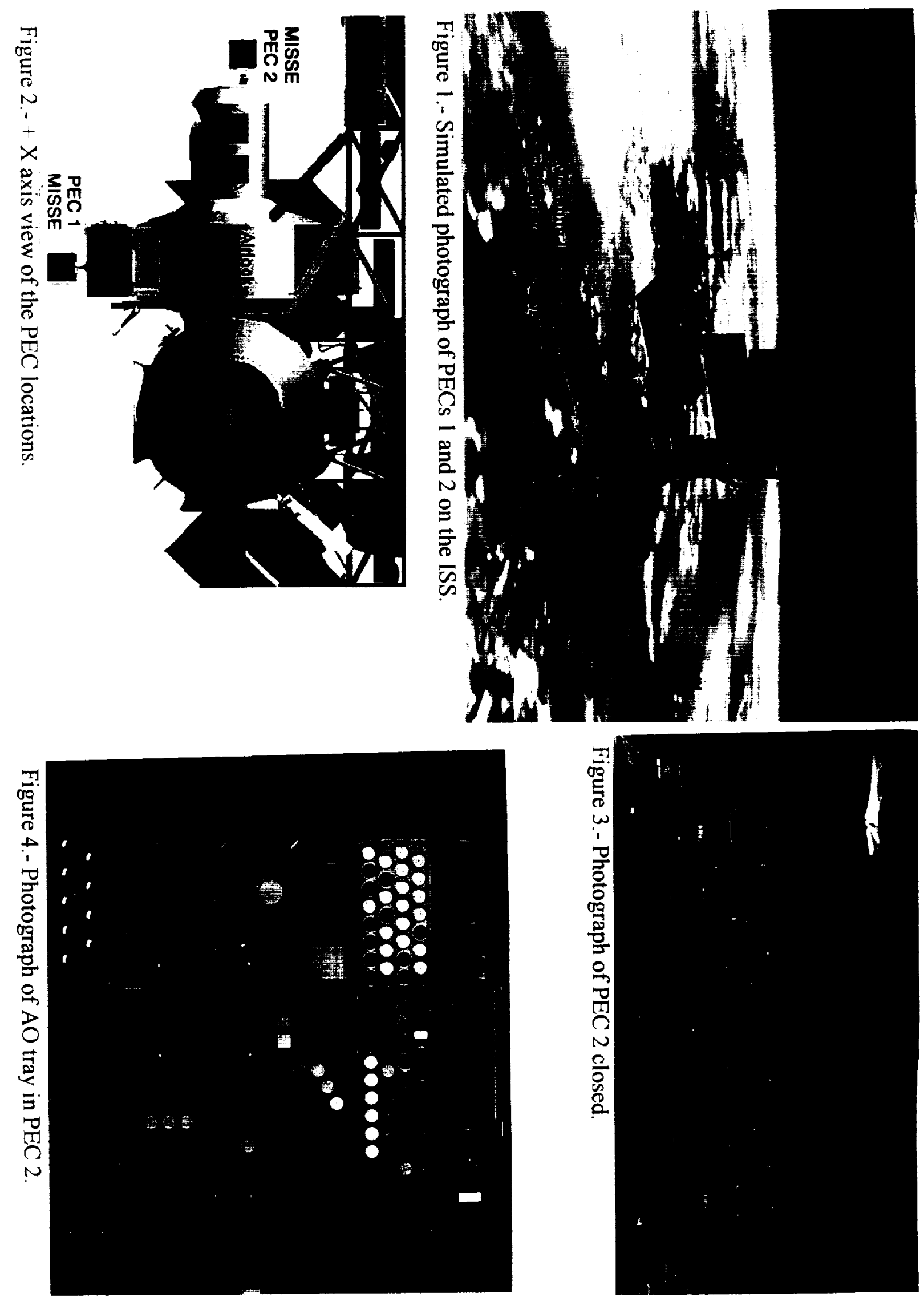
quartz crystal oscillator (QCO) system to obtain molecular mass deposition histories of ISS space exposed hardware.

\section{REFERENCES}

1. Harvey, G. A. , D. H. Humes, and W. H. Kinard, Mir Environmental Effects Payload and Returned Mir Solar Panel Cleanliness. Proc. SAMPE 44, pp1038-1050 (1999)

2. Harvey, G. A., D. H. Humes, and Kinard, Optical Characterization of Returned Mir Solar Cells AIAA 99-4488, Space Technology Conference and Exposition, Albuquerque, NM September 1999.

3. Visentine, J. et. al., Mir Solar Array Return Experiment, AIAA 98-2697, $20^{\text {th }}$ AIAA Aerospace Sciences Meeting and Exhibit, Reno NV, January 1999.

4. Harvey, G. A., D. H. Humes, and W. H. Kinard, Shuttle and Mir Special Environmental Effects and Hardware Cleanliness, High Perform. Polym. 12. pp65-82, 2000.

5. Harvey, G. A., W. H. Kinard, and L. A. Wilson, Outgassing of Flown and Unflown Mir Solar Cells, NASA/CP-2000-209967, Twenty-first Space Simulation Conference, Annapolis, MD, October 2000.

6. Soares, C. E. and R. R. Mikatarian, Mir Contamination Observations and Implications to the International Space Station, Proc of SPIE, Vol 4096, pp55-65 August 2000.

7. Killough, B, CERES TRMM PFM Post-Launch Instrument Performance Thermal Evaluation, unpublished report, 1998

8. Berrios, W. M., Use of the Long Duration Exposure Facility Thermal Measurement System for the Verification of Thermal Models. First Postretrieval Symposium, NASA CP-3134, pp69-83 Kissimmee, FL, June, 1991.

9. Sampair, T. R., A Generalized Approach to the Thermal Analysis of the Long Duration Exposure Facility's Flight Experiments. Second PostRetrieval Symposium, NASA CP-3194, pp27-49. San Diego, CA, June 1992
Table 1

Tabular Simulated-Orbital Temperatures

Date

Time

Temp. ,C

$03 / 21 / 01 \square$

4:00 24:00]

24.21

$03 / 211010$

4:00 39:00]

24.21

$03 / 21 / 01$

$03 / 21 / 01]$

$03 / 21 / 01=$

$03 / 21 / 01$ ?

03/21/01]

$03 / 21 / 01]$

$03 / 21 / 01$

03/21/01]

03/21/01]

03/21/01]

03/21/01]

03/21/01 =

03/21/01

$03 / 21 / 01$

03/21/01 J

03/21/01:

03/21/01 =

03/21/01.

03/21/01 -

$03 / 21 / 01=$

03/21/01=

$03 / 21 / 01$ -

03/21/01 :.

03/21/01]

03/21/01]

03/21/01]

$03 / 21 / 01 \square$

$03 / 21 / 01]$

03/21/01]

03/21/01]

$03 / 21 / 01$

$03 / 21 / 01$

03/21/01]

03/21/01]

$03 / 21 / 01]$

03/21/01]

$03 / 21 / 01 \square$

03/21/01]

03/21/01]

$03 / 21 / 01]$

$03 / 21 / 01 \square$

03/21/01]

03/21/01]

03/21/01]
4:00 54:00]

24.15

5:00 09:00]

24.15

24.15

24.15

24.15

42.68

50.33

55.93

61.15

50.63

46.66

44.1

41.87

54.78

61.23

54.34

48.62

45.79

43.3

51.36

60.59

52.39

47.55

44.79

42.45

51.03

60.33

55.74

48.43

45.61

43.18

43.92

59.45

61.15

48.84

45.91

43.44

41.19

58.31

63.52

49.13

46.15

43.61

41.35 
(Figure 10). All flight data loggers in PEC 2 performed nominally during this heated vacuum exposure and precise temperatures of the MISSE PEC 2 experiment trays were obtained. One of the PEC 2 heated-vacuum-exposure tray-temperature-histories is presented in figure 10.

\section{MISSION DATA ANALYSES AND CORRELATION PLAN}

Temperatures of external hardware are affected by changes in solar illumination during an orbital cycle of approximately 92 minutes. However, the solar illumination per orbit also varies over weeks and months as the ISS orbit precesses around the Earth Therefore there are orbital ( 92 minute) temperature changes which are strongly dependent on the thermal mass and insulation of the hardware, and longer time period ("seasonal") temperature changes dependent on the orbital precession. The orbital inclination of the ISS is 51.6 degrees. The beta angle range is -75 to + 75 degrees when the $z$-axis is aligned toward the sun for power generation by the solar panels, minimum $\mathrm{drag}$, and torque equilibrium attitude (normal XVV TEA orientation). The PECs 1 and 2 will be attached to face into the ram and wake ( + and $-\mathrm{x}$-axis)

directions for maximum and minimum atomic oxygen exposure. Therefore they will experience maximum solar heating at large beta angles.

The nominal orbital period for LDEF was 94 minutes, and the temperature sampling-period by the tape recorder system was 112 minutes. No orbital period data was reported for LDEF. Seasonal (orbital averaged) temperatures at seven locations on and inside the LDEF were compared with THERM model calculations and reported ${ }^{7}$. No temperature data was measured for MEEP and the MIR returned solar array section. Orbital period temperatures were calculated for the MEEP experiment containers, but were not published Time-in-shadow (which correlates inversely with seasonal hardware temperatures) were calculated for $\mathrm{Mir}^{8}$

The temperature sampling-period for the MISSE experiment trays will be 15 minutes, and the orbital period of the ISS is about 92 minutes. The orbit of the ISS precesses very little during ten or fewer orbits, so that temperatures during adjacent orbits (adjusted for the actual orbital period) can be overlaid onto a single composite orbit by increasing the sample time of a later orbit by about 2 minutes. Figure 11 is the orbital temperature history retrieved from a data logger with a thermister attached to a plate subjected to seven simulated solar heating cycles for a hot (XVV Z Nadir attitude at high beta angle) orbit. A composite simulated-hot orbit temperature history (obtained by overlaying the $6 \times 15$ minute data points on a 92-minute orbit is shown in figure 12. Well-defined and precise orbital-temperature-histories for MISSE experiment trays will be obtained in a similar manner. These temperature histories are expected to be approximately sinusoidal of about $15 \mathrm{C}$ amplitude

The seasonal temperature variations are not nearly as dependent on the thermal mass of the hardware as orbital temperature histories. However, the temperatures are expected to vary widely $(\sim 50 \mathrm{C})$ over the precession cycle. Temperature data will be downloaded from the data loggers and transferred to Excel files. The numerical data form the seven simulated hot orbit heating in Excell format is listed in table 1. Temperature files will be posted on the MISSE web site for access by interested researchers PEC number, tray, logger number, and a chronological number will identify these files.

\section{DISCUSSION}

$\because$

Temperature measurements of space-exposed hardware have been limited. This has driven the need to predict (compute) expected extreme temperatures of critical exposed surfaces such as radiators and thermal control surfaces for survivability analyses. External hardware temperatures are strongly affected by spacecraft orientation and operations. Measured temperature histories can be used to confirm changes in spacecraft orientation in many cases, in addition to providing valuable information on materials and hardware. For example, changes in solar radiation absorptivity, and or emmissivity resulting from material degradation of contaminate films usually cause corresponding changes in temperatures of the exposed hardware. This can be used to deduce amounts of contaminate molecular deposition and retention since most molecular contaminates are instantaneously fixed by relatively small amounts of solar ultraviolet radiation and subsequently are darkened by additional solar UV. The ISS is the largest and most complex spacecraft ever assembled Many of the modules are too large to have vacuum cleanliness certifications performed. The number of planned orbital operations, and assembly and supply operations is greater than for any other spacecraft. These circumstances greatly increase the value and utility of external hardware temperature measurements.

A temperature data logger system has been integrated into the MISSE experiment trays. The temperature data loggers used in the MISSE trays are one type of programmable data loggers that can be used in autonomous space systems, which are returned to Earth. LaRC has developed a molecular contamination monitor based on changes in thermal emissitivy to be flown on MISSE for space flight demonstration. LaRC has also developed an active 
complex and costly, even when spacecraft attitude information is available ${ }^{7}$. The ISS is designed to fly in two standard attitudes and three special attitudes because of temperature, docking, and re-boost requirements. The primary flight attitude is with the $\mathrm{x}$-axis in the velocity vector (ram) direction and the $\mathrm{z}$ axis toward the Earth (XVV Z Nadir TEA). The second standard attitude is with the $\mathrm{X}$-axis perpendicular to the orbit plane (XPOP) which allows the solar arrays to track the sun for maximum power generation. Alternatively, measured temperature histories can be used to confirm orientation maneuvers as well as changes in hardware radiative properties.

Temperature data was recorded during the first year of space exposure of LDEF by the THERM system ${ }^{7,8}$. No measured temperature data is available for MEEP, although some temperature predictions were computed Some time-in-shadow data (which correlates inversely with external hardware temperatures) are available for $\mathrm{Mir}^{6}$

\section{DIGITAL TEMPERATURE DATA LOGGERS}

Temperature data loggers are clamped to the bottom (unexposed) side of each MISSE tray (Figure 5). The MISSE tray-temperature data-loggers (Figure 6) have one external thermistor data channel, and a 12 bit analog to digital converter. These data loggers have a nominal temperature recording range of $-50 \mathrm{C}$ to $150 \mathrm{C}$ and a nominal temperature resolution of $0.1 \mathrm{C}$. These data loggers have an expanded non-volatile memory of 70,000 samples for more than 20 years retention without power. The data logger power source is a $3.6 \mathrm{v}$ internal lithium battery with a nominal 10 year lifetime. The clock accuracy is + or - one minute per month.

The data loggers are compatible with a Windows software package that allows the user to configure (program) the data logger, download, display in graphical or tabular form, and save the stored data for export and archiving. The data loggers have selectable sampling rates, instant or delayed sampling start, and full or overwrite storage modes.

The data loggers are housed in injection-molded ABS plastic case $71 \times 53 \times 18 \mathrm{~mm}$ and weigh 62 grams $(2.2 \mathrm{oz})$

\section{ENVIRONMENTAL TESTS}

Several environmental tests of the engineering units of the data loggers were conducted. These were vibration tests to Shuttle payload levels; and vacuum exposure, outgassing, and simulated solar heating tests. Vibration tests of the integrated (completely assembled) PECs 1 and 2 were conducted. Heated ( $60 \mathrm{C}$ ) vacuum outgassing tests of PEC 1 and 2 have also been performed
A five-channel temperature data logger similar to the flight units was subjected to workmanship vibration testing in each of three orthogonal axes on February 8 , 2000. This test was performed on an Unholtz-Dickie T1000 vibration shaker, located in Bldg. 1250 at NASA LaRC. The vibration levels and duration were chosen in accordance with NASA-Std -7001 (Payload Vibroacustic Test Criteria) and NASA-STD - 7002 (Payload Test Requirements). The data logger Y-axis ( $18-\mathrm{mm}$ dimension) random vibration spectrum is shown in figure 7 . The data logger was evaluated at the conclusion of each axis test for functionality and visually apparent damage. The data logger took data at 20-second intervals throughout the testing period. The software "Realtime" function was used to take intermediate readings between each axis-test.

All real-time readings were verified to read the same temperature environment as the data logger. No apparent visual damage was discovered. Although

' post-test-downloaded data was within the expected ranges for all five channels, channels 2 and 3 indicated open thermocouple circuits during testing in two axes. This was attributed to a loose common connection for each of these tests at the connection terminal. These channels read nominally before and after vibration testing.

A flight-level vibration test was performed on the fully integrated PECs 1 and 2 in December 2000. This test was performed on an Unholtz-Dickie model T4000 vibration shaker located in Bldg. 1250 at NASA LaRC. The desired random vibration spectrum for each of the axes is shown in figure 8 . The temperature data loggers in PECs 1 and 2 performed nominally during and after these tests

A 36-hour high vacuum $\left(\sim 10^{-5}\right.$ Torr) survivability test of a temperature data logger was performed in February 2000 . The data logger was subjected to temperatures below freezing for about five hours during this test. $0.315 \mathrm{mg}$ of silicone and ester oils were outgassed from the data logger and recovered from a cold finger during this test.

A 25 day high vacuum survivability and thermal cycle test was performed on a temperature data logger similar to the flight units in March 2000. The data logger was subjected to radiative heating $(-135 \mathrm{~F})$ and cooling $(\sim-50 \mathrm{~F})$ during this test. This data logger had four thermocouple channels, and the thermocouples were placed at different locations on the test hardware The downloaded temperature-histories are presented in figure 9. The data logger performed nominally during and after this test.

Five flight temperature data loggers in PEC 2 underwent a 10-day high-vacuum cleanliness certification in February 2001. The PEC 2 was heated to about $60 \mathrm{C}$ for 7 days in an $8 \times 15$ foot space simulation chamber during this vacuum exposure 